\title{
Soy Intake and Breast Cancer
}

\section{Virgili $E^{1 *}$, Calza $L^{2}$, Testa $E^{3}$ and Emili $R^{3}$}

${ }^{1}$ Department of Outpatient Nutritionist, Porto Sant'Elpidio, Italy

${ }^{2}$ Department of Outpatient Nutritionist, Fermo, Italy

${ }^{3}$ Department of Oncology, Urbino Hospital S. Maria, Italy

\begin{abstract}
At birth, each individual is colonized by different microbes (bacteria, viruses, Eukaryotes and Archaea) composing the MICROBIOTA. It represents a group of organisms with whom the host establishes a continuous and constant relationship from which they both benefit.
\end{abstract}

\section{Keywords: Microbes; Bacteria; viruses; Eukaryotes; Archaea}

\section{Introduction}

Its composition varies depending on different factors that are age, sex, ethnicity, lifestyle, drugs (in particular, antibiotics) and diet. Its most preserved feature is the huge bacteria abundance compared to the other components. Although the microbiota is diffused in all over the organism, about $90 \%$ of it is in the intestine, where the number of different species reaches 500 and the amount (number) of cells per gram of the luminal content reaches 10 [1].

The microbial community and the host live in a condition of balance (eubiosis) whose role is important to maintain health. In the same way, the disruption of this balance (disbiosis), determined by different factors, is strictly correlated to many pathologies. In fact, microbiota alterations are associated to the risk of inflammatory conditions, autoimmune diseases and malignancy. In particular, disbiosis can promote oncogenesis, tumor progression, therapy response and toxicity profiles of chemiotherapic drugs. Although the microbiota stays in the intestine, it exerts both locally and in other further parts of the body; this is possible thanks to the involvement of hormone intermediates, metabolites and immune messengers.

In 2011, Plottel and Blaser were already about the ESTROBOLOME, such as the aggregate of enteric bacterial genes whose products are capable of metabolizing estrogens.

\section{Estrogens are steroid hormones $(\mathrm{C}-18)$ and they originate from the gradual reduction of cholesterol (C-27). Main endogenous oestrogen's are:}

- Estradiol (E1), predominant in non-pregnant women prior to menopause);

- Estrone (E2), predominant after menopause;

- Estriol (E3), highest during pregnancy.

Estrogens E1 and E2 undergo a first passage in the liver, an irreversible hydroxylation in C-2, C-4 or C-16 positions of the steroid ring, whose metabolites vary in term of hormone potency and half-life. Always in the liver, estrogens and their metabolites are combined by glucuronization or sulfunation allowing the excretion in bile, urine and feces.

The estrobolome steps in during this step, because combined estrogens can be uncombined by enteric bacterial $\beta$-glucuronidase and $\beta$-glucosidase, determining their resorption in blood circulation.

In human microbiota, many different $\beta$-glucuronidase genes were described and they refer to different bacterial species existing in the intestine, even if these genes are particularly detected in Bacteroidetes and Firmicutes phyla (the most present in the intestine of an adult).

A high microbial variability ( $\alpha$-diversity) in fecal samples corresponds to an increase of the hormone metabolites compared to parental estrogens; this leads to the hypothesis that differences in hormones metabolism and levels are correlated to a wide number of microbial species in the intestine.

The enzyme $\beta$-glucuronidase and $\beta$-glucosidase activity is induced by high $\mathrm{pH}$ values. Because of this, the colon cancer risk increases, while the correlation with the breast cancer risk was not pointed out.

Another factor influencing the enzyme activity is the diet: an increase of the activity is reported in healthy subjects consuming diets high in fat or protein, whereas fiber consumption decreases the activity [2].

Estrogens are recognized as one of the causal elements in the onset of breast cancer, in particular the hormone receptor positive (HRpositive) one and they have an important role in neoplastic growth. The estrobolome is, then, important, because it is involved in the modulation of endogenous estrogens production and their reabsorption. In this context, mammary glands suffer an "enhanced" estrogens stimulation determining the pathological malignancy expression.

The most common cancer among women is breast cancer. Although many progresses have been made in oncology, unfortunately this type of cancer often becomes resistant to chemotherapy, radiotherapy and hormonal therapies.

Worldwide it is estimated that there are 14 million new cases each year and about 8 million deaths. This disease, which also affects men and trans-genders, is, in most cases, estrogens positive and estrogens stimulate its growth.

Probably genetics is not the main cause of breast cancer, but rather other crucial factors may have a bearing, such as age at first birth, age at the onset of menarche, age at the onset menopause, use of oral contraceptives, race, ethnicity and diet. In particular, diet plays a fundamental role both in the incidence of this type of neoplasia, and in the prognosis and possible recurrences.

*Corresponding author: Virgili E, Department of Outpatient Nutritionist, Porto Sant'Elpidio, Italy, Tel: 3471869657; E-mail: info@edyvirgili.it

Received June 12, 2018; Accepted June 27, 2018; Published July 02, 2018

Citation: Virgili E, Calza L, Testa E, Emili R (2018) Soy Intake and Breast Cancer. J Health Med Informat 9: 316. doi: 10.4172/2157-7420.1000316

Copyright: @ 2018 Virgili E, et al. This is an open-access article distributed under the terms of the Creative Commons Attribution License, which permits unrestricted use, distribution, and reproduction in any medium, provided the original author and source are credited. 
Generally, diet has $30-35 \%$ of influence both in the onset and in the course of any oncological disease. Excess of calories, high quantities of animal fats, refined sugars and alcohol increase the risk of cancer, while antioxidants and fibers contained in vegetables have antitumor effect.

The role of soy, a staple food in Asia, but increasingly used even in Western populations, is highly discussed in this context [3].

Soy and soy based foods are considered healthy, particularly in many Asia-Pacific countries, where soy products have long been consumed.

Soy and soy-related product have been found to help prevent the occurrence of certain types of cancer, such as breast and prostate cancer.

Soy contains isoflavones, or phytoestrogens that can affect breast cancer cells. There are many studies praising the positive properties of soy isoflavones but there are also many others considering these molecules able to promote cancer development. In reality, we should try to understand the molecular mechanisms with which isoflavones modulate the risk of breast cancer, that is how they manage to enter in synergy with estrogens receptors or, on the contrary, how they can act as antagonists for the receptors themselves. Then, there are mechanisms of hormonal signaling in which the isoflavones are involved, independently from the estrogens which cannot be omitted in this context.

Phytoestrogens are plant compounds structurally similar to estrogens and they are categorized in 5 main classes: flavonoids, isoflavonoids, lignans, coumestani and stilbenes [4].

Dietary isoflavones are not only found in soy, but also in beans and lentils. The amount of food isoflavonoids consumed per capita varies from 30 to $50 \mathrm{mg}$ per day for the elderly people in Japan to less than $3 \mathrm{mg}$ in the US and Europe. The traditional Asian diet is a poor diet, which relies on foods derived from the original first-generation soy (such as tofu, tempeh, miso and soy milk), while Western diets rely, in addition to soybeans, on several food sources such as meat, fish, dairy products, eggs and cereals. Moreover, in the Western diet second-generation soy is used, which is very different from the Asian one (we are talking about GMO soy, grown with the use of chemicals and industrially transformed).

Soy isoflavones can bind to oestrogen receptors and therefore can both inhibit and promote the expression of estrogen-sensitive genes. Many studies indicate that the onset of breast cancer is decidedly lower in the Asian populations because people regularly consume many food isoflavones since childhood. Early exposure to soy would seem to be a fundamental element for cancer prevention. Nevertheless, the interest in isoflavones is wide; in fact, they are successfully used also to alleviate menopausal disorders and are an alternative to hormone replacement therapy. In addition, isoflavones would seem to have the ability to activate the immune system and antineoplastic properties such as inhibition of the enzymes necessary for DNA replication, for metastasis and for signal transduction. We know that estrogens cause breast cancer progression and dietary and pharmacological interventions aiming at blocking or reducing oestrogen production are associated with a favourable prognosis in patients with breast cancer. However, there are also many studies that demonstrate an inverse association between frequent consumption of soy isoflavones and breast cancer risk; other studies claim that soy isoflavones do not have any estrogenic effect in humans and conclude that a diet containing soy derivatives can be considered safe even in patients with risk of relapse [5].

Still other studies confirm a chemo-protective and preventive effect on possible relapses in patients with breast cancer. Going deeper, researchers found out that genistein (a soy isoflavone) is able to suppress mammary carcinoma cells, both in vitro and in vivo in mice, and to inhibit both growth and metastasis. Daidzein (other isoflavone) has antioxidant properties and anti-proliferative effects associated with the ability to induce apoptosis and inhibit cell migration.

Isoflavones, in general, are able to inhibit a key enzyme, 17 betahydroxysteroid dehydrogenase, necessary to convert the estrone into its active form: estradiol.

However, many of the antitumor effects of isoflavones occur through estrogen-independent signaling pathways (e.g. genistein is able to inhibit the matrix metalloproteinase transcription involved in metastasis, and it is able to regulate various molecular pathways involving genes such as Bcl-2, Bax, NF-kB, Akt, BRCA, p53 and it downregulates miR-155s associated with breast carcinoma). Unfortunately, these isoflavones are also phytoestrogens and, as we said before, estrogens can induce carcinogenesis. Indeed, these concerns are not groundless and there are many studies that demonstrate, contrary to those listed above, how genistein at different concentrations (some similar to those that could be introduced with the diet) induces neoplastic growth in mice, both in vitro and in vivo, through the modulation of estrogen receptors [6].

One of these studies highlights how dahydetic consumption of food stimulates the growth of breast carcinoma cells by binding to the estrogen receptor. Still other researchers declare the interference between isoflavones and tamoxifen (anticancer drug belonging to the family of selective estrogen receptor modulators), blocking its therapeutic effect. Some researchers correlate genistein with an uncontrolled activation of the cell cycle always through the estrogen receptor. In another study, it is shown that tumor cells treated with genistein are able to evade apoptosis. Lastly, it would seem that genistein is also able to increase the mRNA levels associated with breast carcinoma. Presumably, the genistein, by binding to the estrogen receptor, activates a series of dependent estrogen genes correlated with cell proliferation.

We are, therefore, facing a confused panorama, where the soy isoflavones have opposite effects, both estrogenic and anti-estrogenic.

Some studies implied that isoflavones intake may reduce the risk of developing breast cancer, but some have shown ambiguous results.

We must always consider that in vitro experiments are far from what happens in a living human and that a mouse is not human being. Furthermore, there in not a standard isoflavone diet to take into account, on the contrary, there are many foods containing isoflavones and this makes difficult to compare and analyse data from various studies. Soy is different depending on the type of cultivation (genetically modified or not), on the phytochemical treatments (organic or not) and the foods deriving from soy differ according to the type of processing (artisanal or industrial). Certainly new standardized studies will be needed to clarify the situation. It should be understood even if other foods containing isoflavones (such as beans, asparagus, lentils, etc...) can be safe in an oncological context.

It is also necessary to clarify the right time to ingest isoflavones, because their protective effect seems to occur only during childhood; while in Western women who begin to consume soy in adulthood there are no benefits. Lastly, we must consider the situation of the intestinal microbiota of each individual. Today we speak of "Estrobolome" that is the set of genes of intestinal microbes able to metabolize estrogens, regulating the levels circulating and excreted at the enterohepatic level. Estroboloma microbes produce the enzyme beta-glucuronidase, which can transform estrogens into their active form (estradiol). 


\section{Conclusion}

Circulating estradiol is able to bind to estrogen receptors, influencing all physiological processes depending on the connection between estradiol and estrogen receptor. If the intestine is healthy and in eubiosis - that is to say that the various microbial species are in balance the estrogen produces the right amount of beta-glucuronidase and the estrogen homeostasis is maintained. If intestinal dysbiosis is present, the activity of beta-glucuronidase can be altered with consequent deficiency or excess of free estrogens. The estraboloma may be the key determining the effects of phytoestrogens on the endogenous levels of circulating estrogens [6].

An important aspect related to the intake of soy and its beneficial effects with respect to cancer is timing. In fact, it has been seen that the decrease in the risk of neoplasia is particularly significant in Asian women as they eat soy since childhood. The decreased incidence of breast cancer in Asian could also depend on other protective factors typical of their diet, such as the high consumption of fish rich in omega 3 or spices, such as wasabi.

Another hypothesis made in reference to the timing has been proposed: it would seem that a diet rich in soy in all the periods of life would have an action on the microbiota selecting species with reduced glucuronidase or glucosidase activity, then with reduced deconjugative activity.

Finally, the beneficial effects of soy on ER-positive tumors disappear if this food is added to the diet after the diagnosis of breast cancer. In this case, soy becomes harmful; some studies have shown that isoflavones can mimic estrogen and promote tumor growth.

\section{References}

1. Baker JM, Al-Nakkash L, Herbst-Kralovetz MM (2017) Estrogen-Gut Microbiome Axis: Physiological and Clinical Implications. Maturitas 103: 45-53.

2. Kwa M, Plottel CS, Blaser MJ, Adams S (2016) The Intestinal Microbiome and Estrogen Receptor-Positive Female Breast Cancer. J Natl Cancer Inst 22: 108.

3. Ronis MJ (2016) Effects of soy containing diet and isoflavones on cytochrome P450 enzyme expression and activity. Drug Metta Rev 48: 331-341.

4. Yasuda MT, Sakakibara H, Shimoicorresponding K (2017) Estrogen- and stress-induced DNA damage in breast cancer and chemoprevention with dietary flavonoid. Genes Environ 39: 10.

5. Ziaei S, Halaby R (2017) Dietary Isoflavones and Breast Cancer Risk. Medicines 7: 4

6. Chajès V, Romieu I (2014) Nutrition and breast cancer. Maturitas 1: 1-92. 